\title{
Targeted Drug Delivery and Treatment of Endoparasites with Biocompatible Particles of pH-Responsive Structure
}

\author{
Patrick D. Mathews, ${ }^{* \dagger}$ Ana C. M. Fernandes Patta, ${ }^{\dagger}$ Joao V. Gonçalves, ${ }^{\dagger}$ Gabriella dos Santos Gama, \\ Irene Teresinha Santos Garcia, ${ }^{*}$ and Omar Mertins*,
}

${ }^{\dagger}$ Department of Biophysics, Paulista School of Medicine, Federal University of Sao Paulo, Sao Paulo 04023-062, Brazil
${ }^{\ddagger}$ Department of Physical-Chemistry, Institute of Chemistry, Federal University of Rio Grande do Sul, Porto Alegre 91501-970, Brazil

\section{Supporting Information}

ABSTRACT: Biomaterials conceived for vectorization of bioactives are currently considered for biomedical, biological, and environmental applications. We have produced a $\mathrm{pH}$-sensitive biomaterial composed of natural source alginate and chitosan polysaccharides for application as a drug delivery system via oral administration. The composite particle preparation was in situ monitored by means of isothermal titration calorimetry. The strong interaction established between the macromolecules during particle assembly led to 0.60 alginate/chitosan effective binding sites with an intense exothermic effect and negative enthalpy variation on the order of a thousand $\mathrm{kcal} / \mathrm{mol}$. In the presence of model drugs mebendazole and ivermectin, with relatively small and large structures, respectively, mebendazole reduced the amount of chitosan monomers available to interact with alginate by $27 \%$, which was not observed for ivermectin. Nevertheless, a state of intense negative Gibbs energy and large entropic decrease was achieved, providing evidence that formation of particles is thermodynamically driven and favored. Small-angle X-ray scattering provided further evidence of similar surface aspects independent of the presence of drug. The physical responses of the particles to $\mathrm{pH}$ variation comprise partial hydration, swelling, and the predominance of positive surface charge in strong acid medium, whereas ionization followed by deprotonation leads to compaction and charge reversal rather than new swelling in mild and slightly acidic mediums, respectively. In vivo performance was evaluated in the treatment of endoparasites in Corydoras fish. Systematically with a daily base oral administration, particles significantly reduced the infections over 15 days of treatment. The experiments provide evidence that utilizing particles granted and boosted the action of the antiparasitic drugs, leading to substantial reduction or elimination of infection. Hence, the $\mathrm{pH}$-responsive particles represent a biomaterial with prominent characteristics that is promising for the development of targeted oral drug delivery.

\section{INTRODUCTION}

Efficient in vivo performance of drug delivery systems has received increasing regard with the development of micro- and nanoparticles of specific structural characteristics, allowing for responsive behavior in determined environmental conditions. ${ }^{1-4}$ Actually, the target drug delivery relies on the concept in which a specific drug carrier incorporates the drug, protecting it from undesired degradation, where it is "safely" carried through a deleterious route of administration, reaching the target site and there ideally delivers the drug through sustained release to warrant ideal pharmacological action. Afterward, easy removal of the now "empty" carrier is desired to avoid cumulative disorders or side effects, which may be attained with the use of biocompatible and biodegradable materials. ${ }^{5,6}$ Therefore, the development of biomaterials with responsive structural behavior based on physicochemical characteristics of the whole route of administration plus the target site are needed.

For the development of a drug delivery biomaterial, one ought to primarily consider the route of administration, i.e., intravenous, oral, ocular, transdermal, and so on. Oral administration is assumed to be a better alternative to parenteral administration considering principally the convenience and increased compliance of patients. ${ }^{7}$ Nevertheless, many drugs are degraded or lose their activity during the route of administration and thus before reaching the target at the desired treatment site. For the oral route in particular, one important feature that should not be neglected is the $\mathrm{pH}$ variation. ${ }^{8}$ Indeed, the activity may vary from neutral to strongly acidic or alkaline if considering buccal, stomach, and intestinal tract. With respect to $\mathrm{pH}$ variation, specific $\mathrm{pH}$ responsive biomaterials are under development, resulting in promising and improved therapeutic action of transported drugs. For instance, nanoparticles made with acrylic-based polymers respond to acid $\mathrm{pH}$ of the stomach by carboxyl protonation. ${ }^{9}$ Later, through the gastrointestinal tract when achieving higher $\mathrm{pH}$, hydrogen bond breakage and ionization of the same carboxyl leads to swelling of particles, hence

Received: November 18, 2017

Revised: December 22, 2017

Published: December 28, 2017 
promoting release of encapsulated insulin. Han et al. ${ }^{10}$ developed a more specific $\mathrm{pH}$-sensitive capsule to target the colon and release theophylline. An in vivo study in rats demonstrated that the capsule remained intact from the stomach to small intestine and then disintegrated in the proximal colon, thus optimizing the target drug delivery.

Despite all the progress over the last few years, oral delivery $\mathrm{pH}$-responsive materials require further improvement, especially concerning an increase in the delivery specificity to the site of the drug's expected action, ${ }^{11}$ thus improving the effectiveness of the treatment. Within this concept, all stages involved in the design of the drug delivery system have to be carefully determined for the production of a multifunctional device with known $\mathrm{pH}$-responsive characteristics and prominent site-specific drug targeting. Moreover, in vivo applications require safety of the drug carrier, demanding the use of materials of low immunogenic toxicity, preferred biocompatibility, and easy biodegradation or elimination after achieving the target and expected activity. In this sense, the biomacromolecules alginate and chitosan meet such requirements because both natural source polysaccharides are known to be biocompatible and biodegradable. Chitosan has been studied in diverse drug delivery systems including oral, nasal, intravenous, and transdermal administration. ${ }^{12,13}$ Alginate has received attention due to its excellent gel-forming property, resulting in the development of gel-based drug delivery besides wound dressings. ${ }^{14,15}$ The combination of both polysaccharides has shown further promising applications in the biomedical field, ${ }^{16-18}$ and it relies on the opposite charge profile of the macromolecules in solution with $\mathrm{p} K_{\mathrm{a}} 6.0$ for chitosan and 3.38 (mannuronate residues) and 3.65 (guluronate residues) for alginate, which allows for molecular interactions that may be controlled in specific experimental conditions, leading to the assembly of drug carriers with defined characteristics. Alginate/ chitosan particles may be produced by complex coacervation, where the opposite charges of the biopolymers in solution promote cross-linking and assembly of particles. ${ }^{19-21}$ Nevertheless, the strength of the electrostatic interaction, which provides the final structures and properties, has been poorly described in the assembly process of such drug carrier production.

Within these challenges, in the present article a detailed physicochemical study of a drug delivery particle is primarily reported. Composite alginate-chitosan particles have been produced; however, the interaction between the biomolecules was thermodynamically monitored in situ during the assembly. Moreover, two hydrophobic model drugs, namely mebendazole and ivermectin, of relative small and large molecular structures, respectively, were incorporated into the particles, and the influence and location of both were evaluated. Both drugs are well-known as anthelmintic drugs of negligible water solubility, but they have predicted partition coefficients $(c \log P)$ of 2.95 and 5.83 respectively, characterizing a strong interaction with hydrophobic media. ${ }^{22}$ With these characteristics, the use of drug carriers becomes important to allow and promote delivery to specific sites of action. The $\mathrm{pH}$-responsive behavior of the particles has been monitored over a large $\mathrm{pH}$ range concerning production and application conditions to determine the related structural characteristics.

The target drug delivery, by applying the composite particles via oral administration, was further studied in vivo in the treatment of parasites in Corydoras fish highly infected over the whole gastrointestinal tract. The efficacy of the particles was hence tested with vectorization of the antiparasitic drugs and effectiveness of treatment. Thereby, the development of a $\mathrm{pH}$ responsive drug delivery system of technologic appeal and specific applicability in oral administration is described here.

\section{MATERIALS AND METHODS}

2.1. Materials and Animals. Chitosan ChitoClear 43000 (Pandalus borealis; batch: TM4545) was provided by Primex (Iceland) with 95\% degree of deacetylation (DDA), average molecular weight $M_{\mathrm{w}}=130 \mathrm{kDa}$ (corresponding to 797 repeat monomers per molecule), and low viscosity $(<20 \mathrm{cP})$. For traces of impurities to be eliminated, ${ }^{23}$ chitosan was dissolved at $5 \mathrm{mg} / \mathrm{mL}$ in acetic acid solution ( $50 \mathrm{mM}, \mathrm{pH} 3.0)$. The solution was stirred for $24 \mathrm{~h}$ at 1500 $\mathrm{rpm}$ at room temperature and then filtered through a cellulose acetate Millipore membrane $(0.80 \mu \mathrm{m}$ pore size $)$. After, chitosan was precipitated by the addition of $\mathrm{NaOH}(10 \%$ solution until $\mathrm{pH} 8.5)$ and then filtered (G2 sintered glass) and washed with purified water (Milli-Q) until a conductivity of $4.5 \mathrm{mS}$ in washing water was achieved. Then, it was further washed with water/ethanol solutions (30:70, 20:80, 10:90, and 0:100, v/v), dried, and kept in a silica gel desiccator under a vacuum. Sodium alginate (from brown algae Macrocystis pyrifera) was from Sigma-Aldrich (St. Louis, MO, USA) with $61 \%$ mannuronic acid and 39\% guluronic acid with an average $M_{\mathrm{w}}=200$ $\mathrm{kDa}$ (1143 uronic acid units) and low viscosity (5-40 cP). Chitosan stock solution was prepared by overnight stirring in acetate buffer ${ }^{24}$ $(80 \mathrm{mM}, \mathrm{pH} 4.50 \pm 0.02)$ at $1 \mathrm{mg} / \mathrm{mL}$ and diluted when required. Mebendazole (5-benzoyl-2-benzimidazolecarbamic acid methyl ester; 98\%) was from Sigma-Aldrich, and ivermectin Ivermectan (22,23dihydroavermectin) for veterinary use was provided by Uzinas Chimicas (Jaboticabal, Brazil). All reagents were of analytical grade, and solutions were prepared using deionized water from the Milli-Q Millipore system with a total organic carbon value of less than $15 \mathrm{ppb}$ and a resistivity of $18 \mathrm{M} \Omega \mathrm{cm}$.

Fish were adult Corydoras schwartzi collected from the Amazon basin at Rio Negro River, municipality of Santa Isabel do Rio Negro $\left(0^{\circ} 24^{\prime} 50^{\prime \prime} \mathrm{S}, 65^{\circ} 01^{\prime} 08^{\prime \prime} \mathrm{W}\right)$, Amazonas State, Brazil, as informed by the commercial provider, in November 2016 with a $4.83 \mathrm{~g}$ average weight measuring between 5 and $6 \mathrm{~cm}$ length and transported to the laboratory under appropriate conditions of temperature and water aeration according to Brazilian law. ${ }^{25}$ Upon arrival, fish were equally distributed between five aquariums of $30 \mathrm{~L}$ each previously conditioned to adequate water conditions for the fish with a constant temperature of $28{ }^{\circ} \mathrm{C}$ using thermostat systems (Hopar Aquarium Heater H-606 150W, China). Tap water was dechlorinated, and the $\mathrm{pH}$ was adjusted to 6.50 (Seachem Prime water conditioner, Madison, GA, USA) and submitted to constant filtration and aeration (Aquathec FE25 Filtration System, China) with the flow rate adjusted to $250 \mathrm{~L} / \mathrm{h}$. Fish were fed with commercial ration TetraMin (Tetra GmbH, Germany) adequate for tropical ornamental fish twice a day (8 AM and 5 PM) and monitored for 5 days.

2.2. Preparation of Composite Particles. The alginate-chitosan particles were prepared by titration of alginate solution in chitosan solution under continuous stirring as a modified protocol from Ali et al. ${ }^{26}$ Typically, $1 \mathrm{mg} / \mathrm{mL}$ of alginate solution was prepared by overnight stirring in the same aqueous buffer as chitosan (acetate buffer, $80 \mathrm{mM}, \mathrm{pH} 4.50 \pm 0.02)$. The alginate solution $(50 \mathrm{~mL})$ was slowly titrated drop by drop at $2 \mathrm{~mL} / \mathrm{min}$ in chitosan solution $(1 \mathrm{mg} /$ $\mathrm{mL}, 50 \mathrm{~mL}$ ) under stirring $(1200 \mathrm{rpm})$, and colloidal particles were obtained with a strong electrostatic interaction between the two macromolecules. After complete addition of alginate, the suspension was kept stirring overnight. For the preparation of drug-containing particles, ivermectin or mebendazole, the drug was previously added to chitosan solution, and the mixture was kept stirring overnight (800 rpm). Next, the protocol above was followed. Particles of three different concentrations of each drug were prepared, namely, 38, 15, and $4 \mu \mathrm{M}$. All particle dispersions were centrifuged (Sorvall Super T21 Centrifuge; Newtown, CT) at $21000 \mathrm{rpm}$ and $4{ }^{\circ} \mathrm{C}$ for $20 \mathrm{~min}$. The supernatant was removed, and a new equivalent volume of pure water ( $\mathrm{pH}$ 6.3) was added; the samples were newly centrifuged over $20 \mathrm{~min}$, 
and the supernatant was removed again. Following, all samples were lyophilized (Liotop Liobras, SP, Brazil). For DLS, zeta potential, and SAXS experiments, at the time of the measurement, $10 \mathrm{mg}$ of particles were individually dispersed in $5 \mathrm{~mL}$ of water using a vortex mixer (Gehaka Av-2; SP, Brazil) for 10 s. An aliquot of $100 \mu \mathrm{L}$ of each sample was diluted to $10 \mathrm{~mL}$ in different buffers for the studies of $\mathrm{pH}$ dependence. A buffer of $\mathrm{pH} 2.50$ was prepared using sodium citrate and hydrochloric acid. Buffers of $\mathrm{pH} 3.79,4.10,4.32,4.50$, and 6.50 were all prepared with sodium acetate and acetic acid by varying the proportions of salt and acid to achieve the final desired $\mathrm{pH}$. The maximal variation in $\mathrm{pH}$ was 0.02 . All buffers were at the same concentration of $80 \mathrm{mM}$. Chitosan and alginate solutions were also prepared at $0.1 \mathrm{mg} / \mathrm{mL}$ in five independent samples of each polymer for each $\mathrm{pH}$ for measurements of hydrodynamic diameter of the sole polymers. Solutions were stirred overnight $(1500 \mathrm{rpm})$ at room temperature, and the DLS analysis was performed right after.

For eventual mass loss of the components during particle preparation to be evaluated, triplicates of drug-free and drugcontaining particles for the highest concentration of each drug were prepared as described above, and the supernatants removed by centrifugation were also lyophilized. The recovered dried mass of the supernatants was weighed and subtracted from the initial mass in preparation and corresponded to an average of $16 \mathrm{mg}$. No expressive difference of recovered mass was found between samples comparing drug-free and drug-containing particles (see Table S1); thus, we assume that the recovered mass must correspond to alginate and/or chitosan. For the components of the washing process to be identified, the recovered mass was dispersed in $50 \mathrm{~mL}$ of phosphate buffer $(\mathrm{pH}$ $8.0,80 \mathrm{mM}$ ) and able to solubilize alginate. The mixture was stirred at $1500 \mathrm{rpm}$ overnight at room temperature and then centrifuged as described above; the supernatant was then separated and saved. The pellet was submitted again to the same procedure, and the new supernatant was collected and added to the first one. Supernatants and pellet were lyophilized, and the dry mass weights corresponded to averages of $6 \mathrm{mg}$ for the pellet and $9 \mathrm{mg}$ for the supernatant considering an individual sample. The mass from the pellet was dissolved in $50 \mathrm{~mL}$ of buffer at $\mathrm{pH} 2.5$, and no precipitation was observed after centrifugation, hence, corresponding to chitosan, whereas the other mass corresponded to alginate. Thereby, the correct concentrations of chitosan and alginate in the final particles were 0.92 and $0.82 \mathrm{mg} / \mathrm{mL}$ corresponding to 7 and $4 \mu \mathrm{M}$, respectively.

2.3. Isothermal Titration Calorimetry (ITC). ITC measurements were performed with a VP-ITC microcalorimeter from MicroCal Inc. (Northampton, MA). The working cell of $1.442 \mathrm{~mL}$ in volume was typically filled with a free chitosan acetate buffer solution $(\mathrm{pH} 4.50 \pm$ 0.02 ) or containing mebendazole or ivermectin. The reference cell was filled with the same buffer. For ITC measurements, the chitosan solution free of drug or containing the highest concentration of each drug was diluted to $0.5 \mu \mathrm{M}$ of chitosan with the same buffer, whereas alginate was at $5 \mu \mathrm{M}$. One aliquot of $2 \mu \mathrm{L}$ followed by 27 aliquots of $10 \mu \mathrm{L}$ of alginate solution were injected stepwise with a $200 \mathrm{~s}$ interval into the working cell. The corresponding reference blank experiments were also performed, namely, titration of buffer in chitosan solution and titration of alginate solution in buffer. For avoiding the presence of bubbles, all samples were degassed for $5 \mathrm{~min}$ shortly before starting the measurements. The sample cell was constantly stirred at a rate of 307 $\mathrm{rpm}$, and the measurements were performed at $25{ }^{\circ} \mathrm{C}$. The data analyses were carried out with Origin software provided by MicroCal.

2.4. Small-Angle X-ray Scattering (SAXS). SAXS data were obtained at the magnetic beamline SAS of the Brazilian Synchrotron Light Laboratory (LNLS). The composite particle samples were individually injected in a stainless-steel sample holder, and the temperature was stabilized at $25^{\circ} \mathrm{C}$. The wavelength of the incident beam was $1.605 \AA$, and the linear detector was placed at $43.5 \mathrm{~cm}$ from the sample. An exposure time of $60 \mathrm{~s}$ was applied for intensity acquisitions. The resulting data were normalized to constant beam intensity and corrected for transmission, sample thickness, and parasitic and background scattering according to standard procedures. ${ }^{27}$ Intensity $I$ was plotted as a function of the wave vector $q$, and curves were analyzed in the Porod's region, ${ }^{28}$ as discussed.
2.5. Dynamic Light Scattering. The DLS measurements of particle size and size distribution were performed with a Malvern Zetasizer $300 \mathrm{ZS}$ (Malvern Instruments) operating with a $4 \mathrm{~mW} \mathrm{HeNe}$ laser at a wavelength of $632.8 \mathrm{~nm}$ and detection at an angle of $173^{\circ}$. All measurements were performed in a temperature-controlled chamber at $25{ }^{\circ} \mathrm{C}$. The typical autocorrelation function was acquired using exponential spacing of the correlation time. The data analyses were performed with software provided by Malvern. The intensity-weighted size distribution was obtained by fitting data with a discrete Laplace inversion routine. ${ }^{29}$ Size determination was made using a StokesEinstein relation. ${ }^{30}$ The polydispersity of particles was accessed by using cumulant analysis of the correlation functions measured by DLS applying the amplitude of the correlation function and the relaxation frequency. The second-order cumulant was used to compute the polydispersity of samples. ${ }^{31}$

2.6. Zeta Potential. The zeta potential of particles was measured with the same Malvern Zetasizer performing at least 50 runs per sample at $25{ }^{\circ} \mathrm{C}$. The principle of the measurement is based on laser Doppler velocimetry. The mobility $U$ is converted to zeta potential $\zeta$ using the Helmholtz Smoluchowski relation, $\zeta=U \eta / \varepsilon \varepsilon_{0}$, where $\eta$ is the solution viscosity, $\varepsilon$ the dielectric constant of water, and $\varepsilon_{0}$ the permittivity of free space.

2.7. In Vivo Experiments. Following the fifth day upon fish arrival and conditioning, ten specimens of Corydoras schwartzi were randomly collected one by one from the aquariums using an appropriate collecting net. The fish were euthanized in a benzocaine solution overdose $(400 \mathrm{mg} / \mathrm{L})^{32}$ in accordance with Brazilian law (Federal Law No. 11.794, dated 8 October 2008 and Federal Decree No. 6899, dated 15 July 2009), and all organs and body fluids were examined for parasite infection using an Axioplan 2 Zeiss microscope (Göttingen, Germany) ${ }^{33,34}$ For parasitological exams of stomachs and intestines, the organs were removed and placed in Petri dishes and then examined. $^{35}$

For in vivo experiments, a larger amount of each particle was prepared as described in Preparation of Composite Particles, and the suspensions were submitted to lyophilization (Liotop Liobras, SP, Brazil) to obtain the drug delivery structures in powder form. In this way, oral administration is facilitated because the powder is easily combined with the normal commercial fish ration without changing physical properties of the latter besides stimulating particle intake. After lyophilization, the samples were maintained in the fridge $\left(4{ }^{\circ} \mathrm{C}\right)$ before use. At the time of administration, a weighted amount of each particle was mixed with a weighted amount of the ration, and the fish were fed. The weighted amounts of all administered particles were similar among the aquariums because drug-containing particles were previously prepared with different concentrations of the respective drug. The amount considered the drug dose, which was calculated in $\mathrm{mg}$ per $\mathrm{kg}$ of fish body weight considering the average weight of $4.83 \mathrm{~g}$ of the Corydoras fish. Starting at the seventh day after fish arrival, particles containing the three concentrations of ivermectin and drugfree particles were in this way administrated along the normal morning feeding (8 AM) to fish of four aquariums. The fifth aquarium was the control, and fish were fed the ration alone. After the 5th, 10th, and 15th day of administration, five specimens from each of the five aquariums were randomly collected and analyzed as described above. The same protocol was followed for mebendazole particles with a new lot of fish and after renovation of water of the aquariums. The animal protocols carried out during in vivo studies were approved by the Animal Ethical Committee of the Federal University of Sao Paulo.

2.8. Data Fitting and Calculations. The thermodynamic parameters of alginate binding to chitosan during in situ particle production were obtained from ITC by applying the model for a single set of identical binding sites using a nonlinear least-squares fitting. ${ }^{36,37}$ The model describes the equilibrium between unbound protonated chitosan monomers and unbound ionized alginate monomers. Hence, the binding constant $K$ is defined as

$$
K=\frac{\Theta}{(1-\Theta) C_{\text {free }}}
$$



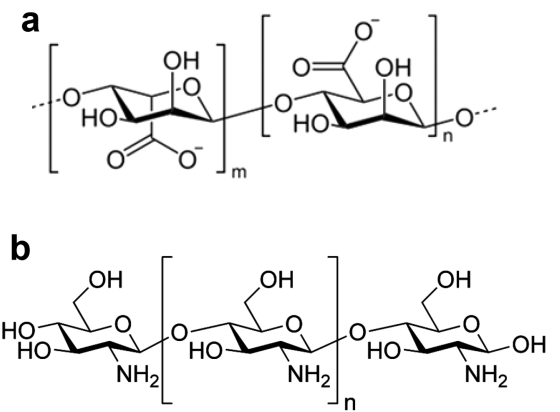

C

d<smiles>COC(=O)Nc1nc2ccc(C(=O)c3ccccc3)cc2[nH]1</smiles>

Figure 1. Molecular structures of ionized alginate containing $m$ guluronic units and $n$ mannuronic units (a), idealized chitosan (b), ivermectin (c), and mebendazole $(\mathrm{d})$.
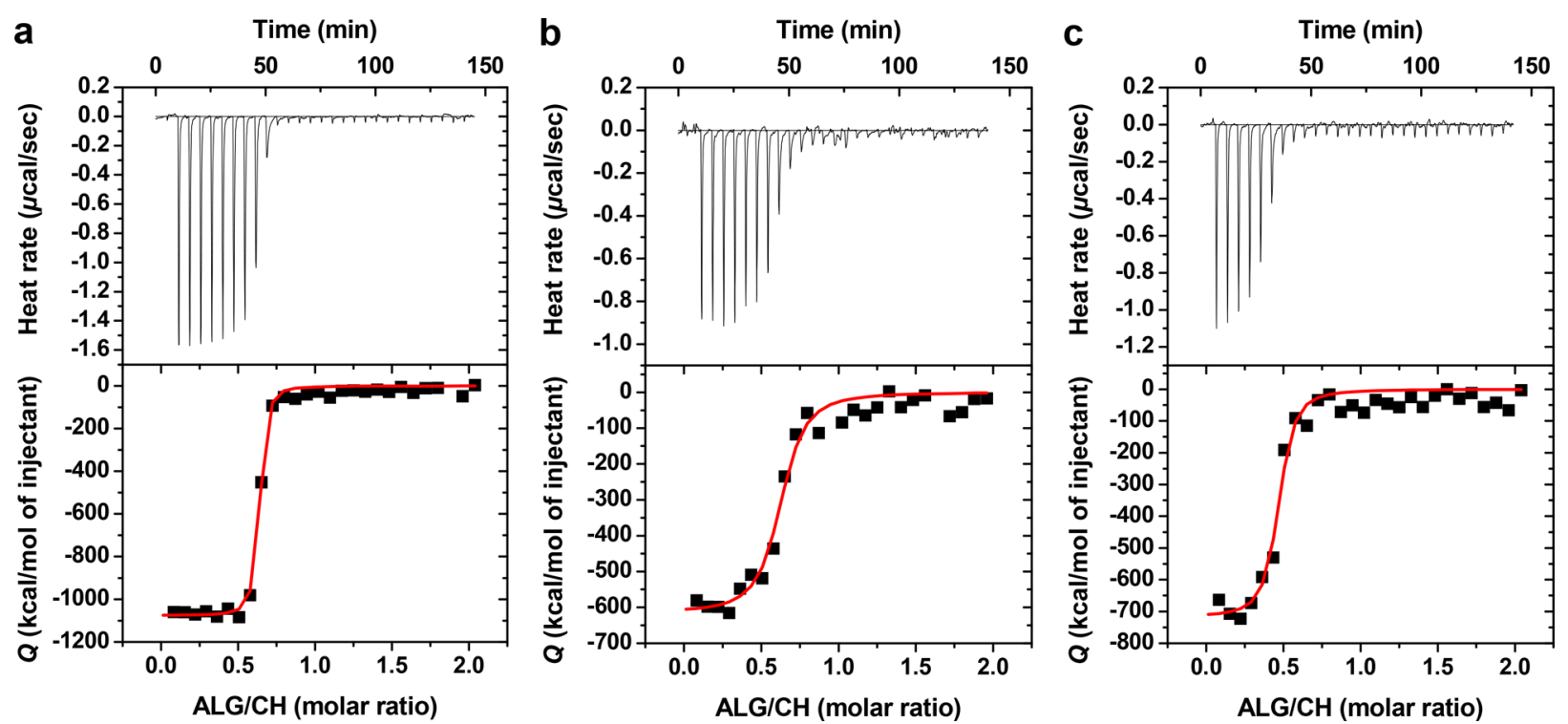

Figure 2. ITC data for the titration of alginate solution (ALG, $5 \mu \mathrm{M}$ ) into chitosan solution ( $\mathrm{CH}, 0.5 \mu \mathrm{M})$ both in an $80 \mathrm{mM}$ acetate buffer at pH $4.50\left(25{ }^{\circ} \mathrm{C}\right)$. The heat flow (upper panels) and the respective integrated binding heats (lower panels) are plotted for particles free of drug (a), particles containing $2.5 \mu \mathrm{M}$ ivermectin (b), and particles containing $2.5 \mu \mathrm{M}$ mebendazole (c). The solid sigmoidal curves in lower panels are fits according to the model for one set of binding sites (see text for details and Table 1 for the corresponding fitting parameters).

where $\Theta=[$ bound alginate $] / N[L]$ is the fraction of chitosan binding sites occupied by alginate monomers, $[L]$ is the total concentration of chitosan-protonated monomers, and the number of binding sites $N$ represents the number of alginate monomers bound to each chitosan monomer at saturation of binding sites. In eq $1, C_{\text {free }}$ is the concentration of free alginate representing

$$
C_{\text {free }}=C_{\text {tot }}-N \Theta[L]
$$

where $C_{\text {tot }}$ is the total monomeric alginate concentration. The combination of eqs 1 and 2 provides a quadratic equation for the molar fraction $\Theta$, and by solving this equation, one gets an expression for $\Theta$ as a function of chitosan concentration. ${ }^{37,38}$ Therefrom, the heat release per injection, $\delta Q$ where the bound fraction changes by $\delta \Theta$, is $\delta Q=N[L] V \Delta H \delta \Theta$ with $V$ the volume in the sample cell and $\Delta H$ the molar enthalpy. By fitting the measured heat release $\delta Q$ with this expression, the fitting parameters $N, K$, and $\Delta H$ are determined (for more details, see refs 36-38).

Considering that chitosan characteristics in solution strongly depend on $\mathrm{pH}$, degree of protonation (DP\%) was calculated considering the respective buffer $\mathrm{pH}$. For determining precise DP\%, two approaches developed by Rinaudo et al. were applied. ${ }^{39,40}$ The first considers complete dissociation of acid, which applies for $\mathrm{HCl}$ buffer solution at $\mathrm{pH} 2.50$. The degree of protonation is obtained via

$$
\mathrm{p} K_{\mathrm{a}}=\mathrm{pH}+\log _{10}[\alpha /(1-\alpha)]
$$

where $\mathrm{p} K_{\mathrm{a}}=6$ for chitosan ${ }^{39}$ and $\alpha$ is DP\%. The second approach considers acetic acid equilibrium of dissociation and applies for acetate buffer solutions of $\mathrm{pH} 3.79,4.10,4.32,4.50$, and 6.50. One considers

$$
\alpha=\left(\alpha^{\prime \prime} \mathrm{C}_{\mathrm{a}}-\left[\mathrm{H}_{3} \mathrm{O}^{+}\right]\right) / \mathrm{C}_{\mathrm{p}}
$$

where $C_{\mathrm{a}}$ and $C_{\mathrm{p}}$ are concentrations of acid and polymer, respectively, $\left[\mathrm{H}_{3} \mathrm{O}^{+}\right]$is calculated from the $\mathrm{pH}$ of the respective buffer solution, and $\alpha^{\prime \prime}$ is degree of dissociation of weak acid in the presence of chitosan

$$
\alpha^{\prime \prime}=K_{\mathrm{a}} /\left(K_{\mathrm{a}}+\left[\mathrm{H}_{3} \mathrm{O}^{+}\right]\right)
$$

where $K_{\mathrm{a}}$ is the dissociation constant calculated using $\mathrm{p} K_{\mathrm{a}}=4.75(25$ ${ }^{\circ} \mathrm{C}$ ) for acetic acid.

\section{RESULTS AND DISCUSSION}

\subsection{Thermodynamic Characteristics and Surface}

Structure. The physical structure of drug delivery systems, which is crucial for effective in vivo performance aiming for high treatment yields with low or nonexistent side effects, is designed in preparation procedures relying on a variety of features such as type and concentration of components, 
Table 1. Effective Thermodynamic Parameters ${ }^{a}$ Characterizing the Interaction between Alginate and Chitosan for Drug-Free Particles (ALGCH) and Respective Drug-Containing Particles at $25^{\circ} \mathrm{C}$ As Indicated

$\begin{array}{cccccc}\text { particle } & N & K\left(10^{8} \mathrm{M}^{-1}\right) & \Delta H(\mathrm{kcal} / \mathrm{mol}) & \Delta G(\mathrm{kcal} / \mathrm{mol}) & T \Delta S(\mathrm{kcal} / \mathrm{mol}) \\ \text { ALGCH } & 0.608 \pm 0.003 & 24.9 \pm 7.24 & -1077 \pm 11 & -15.21 \pm 0.18 & -1061 \\ \text { ALGCH ivermectin } & 0.603 \pm 0.014 & 1.90 \pm 0.65 & -615 \pm 21 & -13.68 \pm 0.85 & -601 \\ \text { ALGCH mebendazole } & 0.441 \pm 0.011 & 3.52 \pm 1.42 & -718 \pm 26 & -14.05 \pm 0.89 & -427 \\ { }^{a} \text { Number of binding sites, } N \text {; binding constant, } K \text {; molar enthalpy, } \Delta H \text {; Gibbs energy, } \Delta G \text {; entropic contribution, } T \Delta S \text {. }\end{array}$

solubilization procedures, conditions of component mixing, interactions between structuring molecules, and so forth. Herein, two biocompatible polysaccharides, namely alginate and chitosan (Figure 1), were employed in the production of antiparasitic drug delivery particles. Being strongly and oppositely ionized macromolecules in the $\mathrm{pH}$ range around $4.50,{ }^{41,42}$ both polymers establish electrostatic interactions leading to assembly of the final drug delivery particles as two main component composites. Hence, the intensity of the interaction certainly designates the fate of this kind of particle in terms of its final physical structure. To quantitatively verify the assumption, we performed isothermal titration calorimetry (ITC) in which the conditions of the experiment were similar to the conditions of the particle preparation procedure, i.e., the alginate solution is slowly titrated in chitosan solution with or without antiparasitic drugs under continuous stirring and constant $\mathrm{pH}$ and temperature.

Figure $2 \mathrm{a}$ shows ITC results for the interaction between alginate and chitosan in the production of drug-free particles. The sharp down-pointing peaks (upper panel) represent the heat released at every injection and thus provide evidence of a strong exothermic effect when adding alginate solution to chitosan solution at constant $\mathrm{pH} 4.50$. If comparing results of drug-free particles (Figure 2a) with ivermectin particles (Figure $2 \mathrm{~b}$ ) and mebendazole particles (Figure $2 \mathrm{c}$ ), reduction of heat released at every alginate injection into the calorimeter cell containing chitosan and drug is noticeable. The integrated heats resulting from interaction as a function of alginate/chitosan molar ratio (lower panels) show sigmoidal behavior because with every following injection the heat signal decreases, denoting partial neutralization of protonated chitosan with negatively charged alginate. At every following alginate injection, there is less free chitosan available to interact, and thus, the energy release with the next injection is also a little lower, and so on. The heat released from the interaction ceases when all chitosan in the titration cell is complexed and neutralized with alginate; then, only heats of dilution are observed, which have been subtracted from the final thermodynamic analysis in this study, as described elsewhere. $^{36,43}$ Overall, the strong heat released in the titration is indicative of an energetically favorable interaction between the two macromolecules. Therefore, ITC measurements provide a binding equilibrium constant and enthalpy variation due to the electrostatic interaction during in situ particle preparation.

For analyzing the interaction, thermodynamic parameters were determined with ITC measurements employing the model for a single set of binding sites (see Materials and Methods) with a nonlinear least-squares fitting using Origin software. ${ }^{36,43}$ This model describes the equilibrium between the free protonated chitosan monomers plus free ionized alginate monomers and the bound alginate/chitosan complex. The fitting of measured heat released actually produces the sigmoidal curves in Figure 2 and provides fitting parameters as the number of binding sites $N$, equilibrium constant $K$, and enthalpy variation $\Delta H$. From $K$, one calculates the Gibbs energy related to the interaction applying $\Delta G=-R T$ n$(55.55 \mathrm{~K})$, where $\Delta G$ is defined for standard state of mole fraction, $T$ is temperature, and 55.55 introduces the concentration of water. Therefrom, the entropy gain is further estimated via $T \Delta S=\Delta H-\Delta G$.

The fitting parameters along the calculated Gibbs energy and entropy gain are shown in Table 1. Accordingly, the number of binding sites $N$, which may be translated as number of ionized alginate monomers interacting with protonated chitosan monomers at saturation of binding sites, i.e., the point where chitosan monomers are no longer available to interact is near 0.60 for drug-free and ivermectin particles. This value means $1 /$ $N \sim 1.7$, which provides the ratio of accessible chitosan monomers to bind on alginate monomers at saturation. Intriguingly, the ratio is closer to 2 chitosan monomers for 1 alginate monomer instead of a 1:1 relation. As a matter of fact, in terms of monomer structure dimensions, for chitosan one estimates a monomer-to-monomer charge distance of $\sim 0.52$ $\mathrm{nm},{ }^{37}$ and for alginate the distance between adjacent bridge oxygens (see Figure 1a) is $\sim 0.55 \mathrm{~nm}$; ${ }^{44}$ thus, they present near dimensional features, which in the simplest interpretation would lead approximately to a 1:1 ratio at saturation. Moreover, in terms of macromolecular structure, there is an expressive difference in average molecular weight between alginate, 200 $\mathrm{kDa}$, and chitosan, $130 \mathrm{kDa}$, meaning 1143 and 797 average monomers for each chain of the polymer, respectively. In a hypothetic optimized situation, one would expect one alginate chain interacting with one chitosan chain, which provides 1.43 alginate monomers to 1 chitosan monomer. Nevertheless, the experimental $N \sim 0.60$ evidence is far from an ideal condition, which must be related to molecular conformations of both polymers in solution. Indeed, despite the similarity in basic chain geometry (cellulose type), chitosan is characterized as less extended than alginate. ${ }^{45}$ Hence, the higher flexibility of chitosan leads to conformation changes in solution producing wormlike structures. ${ }^{46}$ Thereby, the strong interaction with extended alginate leads to the proportion of $\sim 1.7$ chitosan monomers per alginate monomer at saturation. A higher difference was obtained for mebendazole particles with $N \sim$ 0.44 (Table 1 ); thus, $1 / N \sim 2.2$. This result provides evidence that the presence of mebendazole increases the amount of chitosan monomers per alginate if compared to those of drugfree and ivermectin particles. On the other hand, the number of effective binding sites $N$ was reduced, meaning that a reduction in the number of protonated chitosan monomers occurred because binding sites depend on effective charge-charge interactions between $\mathrm{COO}^{-}$and $\mathrm{NH}_{3}{ }^{+}$. Presumably, the drug hydrophobic molecules of relatively small structural dimensions (Figure 1d) may be well distributed along the chitosan chains to establish hydrophobic interactions with the polymer backbone. Additionally, partial deprotonation of chitosan may occur when interacting with mebendazole considering competition of protons between chitosan amine and the 
mebendazole ring nitrogens $\left(\mathrm{p} K_{\mathrm{a}}\right.$ 6.6). Thus, the chitosan structural changes proportioned by mebendazole results in an effective increase to $\sim 2.2$ chitosan monomers for 1 alginate monomer at saturation, but the effective number of binding sites $N$, i.e., interaction between the ion pair, decreased $27 \%$ in the presence of mebendazole relative to the other two particles. Compared to ivermectin, this later drug is of relatively larger molecular dimensions (Figure 1c), besides the robust bulky structure, and one may speculate that drug-drug van der Waals and hydrophobic interactions possibly prevailed over a drugchitosan hydrophobic interaction, leading to lower distribution of individual ivermectin molecules along the polymer backbone and instead promoting localized drug aggregates, thereby contributing to $N$ maintenance as for drug-free particles.

The fitting function for heat release that is the sigmoidal curve (Figure 2) also provides binding constant $K$ and molar enthalpy $\Delta H$ for particle production (Table 1). Now, the binding constant $K$ is much larger for drug-free particles compared to that for ivermectin and mebendazole particles. Therefore, the binding strength is indeed higher in the absence of drug, confirming a strong electrostatic interaction between the two polysaccharides. The same conclusion accounts from molar enthalpy, where a significantly negative $\Delta H$ on the order of a thousand $\mathrm{kcal} / \mathrm{mol}$ for drug-free particles denotes an intense exothermic effect during the interaction. Nevertheless, both drug-containing particles still present highly negative $\Delta H$, providing evidence that the formation of all particles is thermodynamically driven and favored.

The calculated Gibbs free energy $\Delta G$ is similar for the three particles (Table 1), indicating that despite $\Delta H$ differences between drug-free and drug-containing particles, all systems achieve a state of intense negative Gibbs energy, denoting that the alginate-chitosan interaction effectively leads to particle formation in the presence of either drug. Furthermore, the entropy contribution $T \Delta S$ is around $2 \times$ more highly negative for drug-free particles despite also being significantly negative for drug-containing particles. The large entropic decrease in the formation of particles reinforces the strong interaction between the macromolecules, where free ionized and extended polysaccharide chains interact through electrostatic forces leading to assembly of composite particles.

Noteworthily, heat contribution due to changes in alginate and chitosan conformation during interaction and assembly of the particles must further contribute to thermodynamic characteristics as discussed above. In particular, if considering drug-containing particles, the comparatively less negative enthalpy variation observed must rely on reduced molecular flexibility of chitosan that is already bearing the drugs. Therefrom, the conditionally increased stiffness of chitosan chains may reduce to some degree the heat release in the complexation process. Other effects including the hydrophobic interaction between polymer backbones, hydrogen bonding, deprotonation of chitosan amines, alteration in ion distributions, and changes is water structure may contribute some to heat release and some to heat absorption.

Nevertheless, the still highly negative thermodynamic parameters obtained for all particles provide evidence overall of a strong electrostatic interaction, which promotes the particle formation even in the presence of large hydrophobic molecules of antiparasitic drugs. The physical structure of the produced particles, discussed in detail in the next section, was preliminary evaluated in terms of surface aspect by means of SAXS.
Figure 3 shows SAXS patterns of the same final particles discussed above. In the double logarithmic plot of scattering

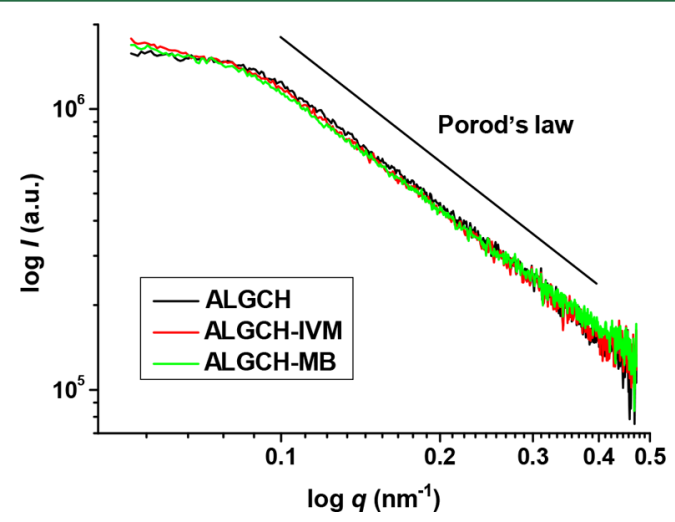

Figure 3. Double-logarithmic plot of the SAXS patterns of intensity $I$ as a function of scattering vector $q$ for the drug-free particles (ALGCH), ivermectin-containing particles (ALGCH-IVM), and mebendazole-containing particles (ALGCH-MB). A shifted curve slope (solid line) is shown for the Porod's region.

intensity $(I)$ as a function of scattering vector $(q)$, it is evidenced that the curves follow Porod's power law in the region upward of $0.1 \mathrm{~nm}^{-1}$, meaning that intensity $I(q)$ is proportional to $q^{-4}$ for larger values of $q$, as predicted for ideal spheres and surface fractals. ${ }^{28,47}$ The data also show a constant additive background resulting from three-dimensional electron density fluctuations in higher $q$ region. Of note, $I(q)$ curves do not present any distinct features; instead, they exhibit practically overlaid scattering patterns with constant slope in the Porod's region. The simple assumption of Porod's law for surface scattering $I(q) \sim S q^{-4}$ denotes that surface area $S$ for all of the particles is constant, which is evidence of a similar surface aspect for the same.

The similarity in the surface aspect of the particles further reinforces the observation that, independent of the presence of the two different antiparasitic drugs studied herein, the particles are produced equally as a result of strong electrostatic forces leading to a state of lower free energy thus with favored thermodynamic conditions and providing drug delivery particles with the same physical surface aspect.

3.2. Structural Dependence on $\mathbf{p H}$. Application of oral drug delivery micro- or nanoparticles requires that these devices further ensure physical responses in various fluids of specific characteristics, which may allow stability during the route of administration while permitting release of transported active compounds in targeted organs, e.g., the gastrointestinal tract. ${ }^{48}$ During the route of oral administration, the $\mathrm{pH}$ varies drastically, and hence, the drug delivery particles may be developed to purposely respond to this variation. Therefore, our particles were submitted to a range of $\mathrm{pH}$ levels in buffer solutions to evaluate physical characteristics.

Figure 4a shows DLS results for a set of composite chitosanalginate particles as a function of $\mathrm{pH}$ variation. Particles containing three concentrations of each drug, i.e., ivermectin or mebendazole, were analyzed along with the control drug-free particle. As shown, all particles presented strong $\mathrm{pH}$ dependence concerning hydrodynamic diameter. At $\mathrm{pH} 2.50$, the particles show large average diameters on a micrometer scale. Interestingly, when increasing the $\mathrm{pH}$ to 3.79 , a size reduction is evident for all particles but more abruptly to the drug-free particle. The same behavior is noted at $\mathrm{pH} 4.10$, where all 

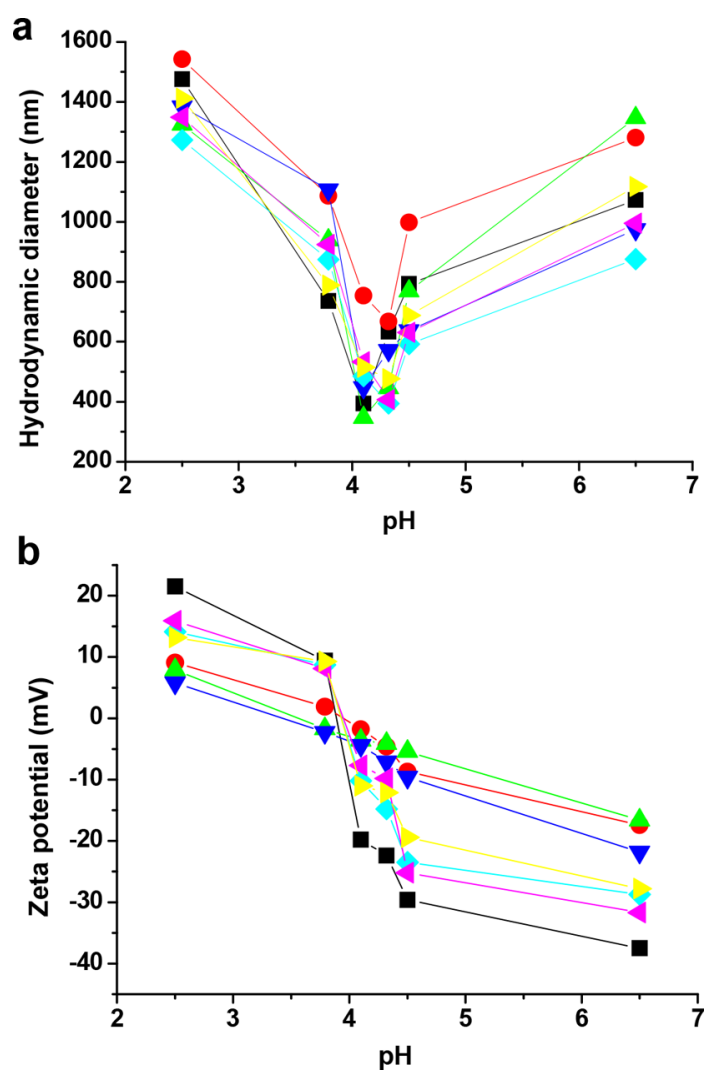

Figure 4. Hydrodynamic diameters (a) and zeta potential (b) as a function of $\mathrm{pH}$ for drug-free particles ( $\mathbf{\square})$, particles containing 4 (red $\boldsymbol{0}$ ), 15 (blue $\boldsymbol{\nabla}$ ), and $38 \mu \mathrm{M}$ (green $\boldsymbol{\Delta}$ ) of ivermectin, and particles containing 4 (magenta 4 ), 15 (cyan $\bullet$ ), and $38 \mu \mathrm{M}$ (yellow $>$ ) of mebendazole. Solid lines are a guide to the eye.

particles assumed an average size on the nanometer scale. Similar behavior is shown at $\mathrm{pH} 4.32$ with a slight increase in the average size. At $\mathrm{pH} 4.50$, the average sizes show a new increment, and finally at $\mathrm{pH} 6.50$, all particles present submicrometric or micrometric diameters but with smaller averages if compared to the initial $\mathrm{pH} 2.50$ for the majority of samples. The polydispersity of all samples was large and on the order of 0.32 and 0.71 for all samples, indicating a large size distribution of the composites. However, when analyzing the average diameters, size dependence on $\mathrm{pH}$ is evidenced and suggests structural peculiarities related to physical stability, ionization, protonation, and hydration of the macromolecules. This assumption is corroborated by the behavior of sole alginate and sole chitosan in diluted solutions $(0.1 \mathrm{mg} / \mathrm{mL})$ of the various $\mathrm{pHs}$, where hydrodynamic diameters of each polymer show strong dependence on $\mathrm{pH}$ (see Table S2). Actually, sole chitosan presents a reduced size in the $\mathrm{pH}$ range of 2.50-4.50 denoting good solubility of the polymer chains but aggregates at $\mathrm{pH} 6.50$ as a result of deprotonation. ${ }^{38}$ Instead, alginate shows smaller size at $\mathrm{pH}$ values between 4.10 and 6.5 , whereas aggregates at $\mathrm{pH} 2.5$ evidence the formation of alginic acid of low water solubility. ${ }^{23}$

Additionally, zeta potential results depicted in Figure $4 \mathrm{~b}$ show a conformable tendency of the particles' structural dependence on $\mathrm{pH}$. At $\mathrm{pH} 2.50$, all particles present zeta potential around neutral to positive values with the drug-free particle bearing the highest $+22.3 \mathrm{mV}$ on average. At $\mathrm{pH} 3.79$, a slight decrease in zeta potential is noticed, and a similar tendency follows for $\mathrm{pH} 4.10,4.32,4.50$, and 6.50 . Actually, the drug-free particles show an abrupt reversal from approximately $+10 \mathrm{mV}$ at $\mathrm{pH} 3.79$ to approximately $-21 \mathrm{mV}$ at $\mathrm{pH} 4.10$, plus decreasing to around $-38 \mathrm{mV}$ at $\mathrm{pH}$ 6.50. Samples containing mebendazole show approximately similar results with nonsignificant drug concentration dependence. On the other hand, particles with invermectin have shown a relatively discrete reduction of average zeta potential as a function of $\mathrm{pH}$ increase, suggesting a drug contribution to the particle surface charge profile for the three studied concentrations. Maximal standard deviation for zeta potential was $4.3 \mathrm{mV}$, and average conductivity was $6.38 \mathrm{mS} / \mathrm{cm}$ for all samples.

As evidenced in Figure 4, both DLS and zeta potential results show that particles feature $\mathrm{pH}$-dependent characteristics. In strongly acidic conditions, the composites present larger size and a neutral-to-positive zeta potential. These results may be related to physicochemical properties of the macromolecules, especially to alginate. Indeed, alginate with $\mathrm{pK}_{\mathrm{a}} 3.38$ (mannuronate $\mathrm{M}$ residues) and 3.65 (guluronate $\mathrm{G}$ residues), is in the hydrated form in strong acid with protonation of carboxylic groups leading to alginic acid of low water solubility. ${ }^{23}$ Hydration of large chains of the polysaccharide leads to an increase in the hydration volume, which thus may increment the hydrodynamic diameter of the particles producing swollen expanded structures as observed and represented in Figure 5. Moreover, chitosan remains ionized

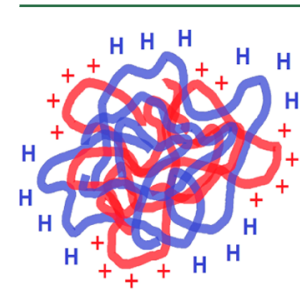

$\mathrm{pH} \sim 2.5$ - 3.8

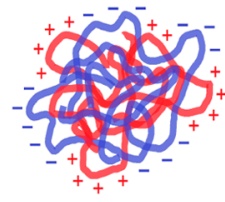

$\mathrm{pH} \sim 4.1$ - 4.5

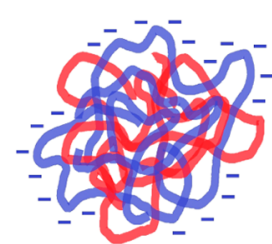

$\mathrm{pH} \sim 6.5$
Figure 5. Representation of alginate-chitosan composite particles. In a strongly acidic solution at $\mathrm{pH} 2.5-3.8$, alginate chains (blue) are neutralized by hydrolysis, whereas chitosan chains (red) present a high degree of protonated amine groups, thus positive charges; these conditions produce a swollen expanded and positively charged structure. In a mildly acidic solution at $\mathrm{pH} 4.1-4.5$, both alginate and chitosan are oppositely charged, and a strong electrostatic interaction produces a compact size-reduced structure. In a slightly acidic solution at $\mathrm{pH} 6.5$, chitosan is neutralized, but highly ionized alginate leads to swelling by anionic self-repulsion, producing a larger and negatively charged structure.

Table 2. Calculated Percentage of the Degree of Protonation (DP\%) for Chitosan Amine Groups over a Range of $\mathrm{pH}$ Levels for Different Buffer Solutions

\begin{tabular}{ccccccc}
$\mathrm{pH}$ & 2.50 & 3.79 & 4.10 & 4.32 & 4.50 & 6.50 \\
$\mathrm{DP}(\%)$ & 100 & 100 & 98.8 & 97.9 & 96.9 & 21.3 \\
\hline
\end{tabular}

at $\mathrm{pH} 2.50$ by complete protonation of amine groups (Table 2), hence leading to particles of positive surface charge, as determined. Because particles were prepared with similar concentration of chitosan and alginate $(\mathrm{w} / \mathrm{w})$, the fact that zeta potential remains slightly positive or close to neutrality, instead of strongly positive, may suggest that chitosan is mostly overlaid by hydrated alginate or partially neutralized by the 
same. Indeed, the proper hydration of alginate must have contributed to the particles' partial neutralization. Thus, in the absence of surface charge, aggregation of particles may also occur besides contributing to the increase in size.

At $\mathrm{pH}$ 3.79, all particles presented somewhat of a size reduction but still relatively large particles. Under these conditions, the $\mathrm{pH}$ is only slightly above the $\mathrm{p} K_{\mathrm{a}}$ of alginate guluronate residues; thus, partial hydration of alginate retains similar structure conditions as for the strongly acidic medium, as discussed above. The protonation degree of chitosan is also not affected at this $\mathrm{pH}$ (Table 2).

More pronounced changes were noted at $\mathrm{pH}$ from 4.10. In fact, a reasonable size reduction along with charge reversal for the majority of samples was determined, indicating that this $\mathrm{pH}$ condition is ideal for the production of particles of lower size. From $\mathrm{pH} 4.10$ to 4.50 , both alginate and chitosan are strongly ionized, i.e., alginate is negatively charged on carboxylic groups, and chitosan is almost fully protonated on amine groups with very slight protonation degree reduction along this $\mathrm{pH}$ range (Table 2). Thereby, an ionized particle of lower size (Figure 5) is obtained, and one may suggest the maintenance of a strong electrostatic interaction between alginate and chitosan in the particle, as determined and discussed in the previous section. The fact that zeta potential is highly negative for the drug-free particles in the $\mathrm{pH} 4.10-4.50$ range, if compared to the majority of drug-containing particles, reinforces the assumption that alginate mostly overlays chitosan, and hence, the negative charges of the former prevail on surface charge distribution. Additionally, the larger average molecular weight of alginate compared to that of chitosan with 1143 and 797 average monomers per chain, respectively, as discussed before further argues in the sense that extended chains of alginate may prevail over shorter chitosan in the complexation during particle assembly, leading to the formation of structures mainly overlaid by alginate.

At the final $\mathrm{pH}$ of 6.50 , a new size increment was observed for all samples along with further reduction of the zeta potential. Under these conditions, chitosan is predominantly neutralized, i.e., with a low degree of protonation on the order of $21 \%$ (Table 2,) whereas alginate remains strongly ionized. In the absence of counterions, the negative charges of alginate now develop intermolecular self-repulsion in the particles, which promotes bulk expansion leading to a size increase as denoted and represented in Figure 5.

Because the majority of zeta potential results are not very far from neutrality, some aggregation may occur in all samples. However, comparing Figure $4 a$ and $b$, when the zeta potential is closer to neutrality for a part of the samples, e.g., samples containing ivermectin, lower size was observed for the same at the corresponding $\mathrm{pH}$. This further corroborates that the main process governing particle characteristics is in fact related to ionization and hydration of the macromolecules. The observation that ivermectin's presence in the composites diminishes zeta potential variation as $\mathrm{pH}$ varies, as compared to mebendazole or drug-free particles, may be somewhat related to the large molecular structure of this drug, thus reducing the effective surface charge of the particles. Besides the differences between the average molecular weights of the polymers, one has to take into account the order of component mixing in the particle preparation. The fact that drugs are previously mixed with chitosan, which is necessary to allow for drug-chitosan association due to hydrophobic characteristics of drugs, increases the bulky structure of chitosan but without threatening the interaction with alginate as shown in ITC experiments. Following, the titration of alginate solution in the chitosan-drug solution leads to particles with a predominance of alginate over surface and chitosan-drug in the core because SAXS suggests the same physical surface aspect for all particles independent of drug absence or kind of drug. Finally, zeta potential of ivermectin particles is less influenced with $\mathrm{pH}$ variation, further suggesting a scenario where chitosan is more internalized in the particle with the bulky drug, and thus, ionized carboxylic groups of alginate tend to face the core of particles to interact with chitosan-protonated amines. This scenario would lead to particles of effective weak surface charge over the various $\mathrm{pH}$ ranges as determined for ivermectin particles. Actually, this particle profile where the drug is predominantly localized in the particle core may account for protection during storage and the route of administration, e.g., protecting the drug from undesired degradation, and can furthermore alter pharmacokinetics and pharmacodynamics of the drug delivery.

3.3. In Vivo Performance. Aiming to evaluate the practical performance of the drug delivery composite particles, we performed in vivo experiments in which the particles were systematically administered via oral administration to Corydoras schwartzi fish collected from the Amazon basin (Figure 6a).

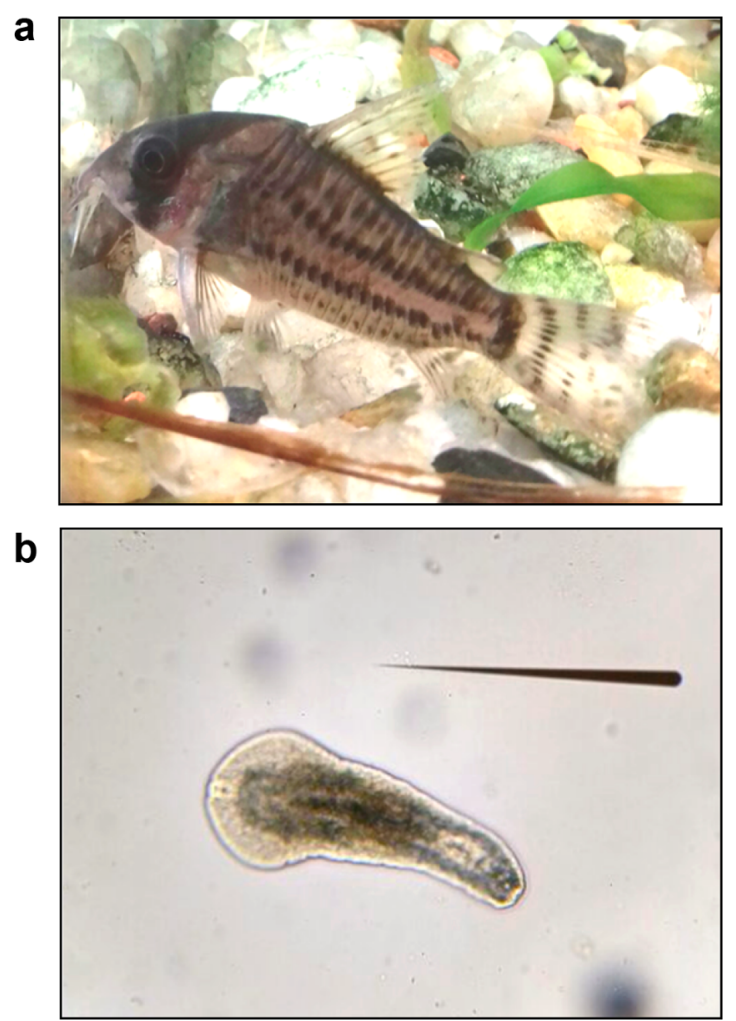

Figure 6. Snapshot of a Corydoras schwartzi fish (a) collected from the Amazon basin and an isolated digenetic trematode (b) found in the fish digestive tract (scale bar spans $500 \mu \mathrm{m}$ ).

Before the experiments, the sampled fish were analyzed a few days after arrival, as detailed in the Materials and Methods, and they were highly infected with nematodes (genus Procamallanus) and digenetic trematodes (Figure 6b) in the whole digestive tract. The parasites were found in the adult stage for nematode and metacercariae for digenetic trematode, and prevalence was $100 \%$ for both (Figure $7 \mathrm{a}$ and c). No other kind 

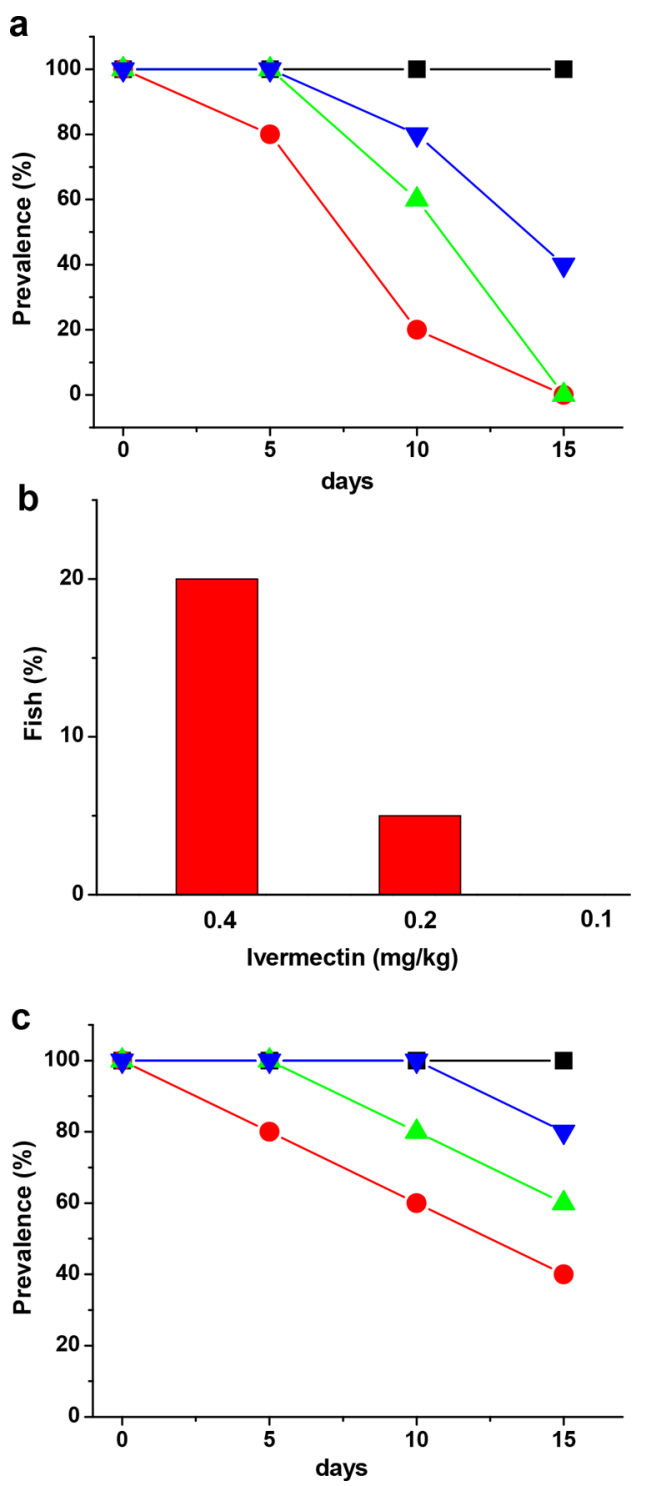

Figure 7. Prevalence of gastrointestinal nematodes in Corydoras schwartzi fish during 15 days of treatment using ivermectin-containing alginate-chitosan composite particles with $0(\mathbf{\square}), 0.1$ (blue $\boldsymbol{\nabla}), 0.2$ (green $\mathbf{\Delta}$ ), and 0.4 (red $\mathbf{0}$ ) $\mathrm{mg}$ of ivermectin per $\mathrm{kg}$ of average fish body weight (a). Percentage of fish mortality occasioned by ivermectin concentrations during parasitosis treatment (b). Prevalence of gastrointestinal digenetic trematodes in Corydoras schwartzi fish during 15 days of treatment using mebendazole-containing alginate-chitosan composite particles with $0(\boldsymbol{\square}), 0.1$ (blue $\boldsymbol{\nabla}$ ), 0.2 (green $\mathbf{\Delta}$ ), and 0.4 (๑) $\mathrm{mg}$ of mebendazole per $\mathrm{kg}$ of average fish body weight (c).

of parasite was found by examining the whole body of the fish. The experiments consisted of feeding the fish daily with lyophilized particles once a day together with the normal morning feeding.

In the first experiment, ivermectin particles with three concentrations of drug were individually administered to fish of three aquariums and drug-free particles to fish in the fourth aquarium. The fish of the fifth aquarium were fed without particles as a control (see Materials and Methods for details). Sampled fish from each aquarium were analyzed at the 5th, 10th, and 15th day of treatment, and the analysis focused on nemotode treatment because ivermectin is ineffective against trematodes due to the absence of high-affinity binding sites. ${ }^{49}$
As shown in Figure 7a, parasite prevalence remained at $100 \%$ equally for the control and drug-free particles over the whole experiment. This result provides evidence that drug-free particles themselves do not provide treatment as expected. Moreover, the same drug-free particles did not alter any visible physical aspect or behavior of the fish as comparing to those of the control. Regardless, ivermectin particles showed systematic reduction of parasite prevalence: the lower concentration (0.1 $\mathrm{mg} / \mathrm{kg}$ ) provided decreases to $80 \%$ (10th day) and $40 \%$ (15th day); the intermediate concentration $(0.2 \mathrm{mg} / \mathrm{kg})$ provided decreases to $60 \%$ (10th day) and $0 \%$ (15th day), and the higher concentration $(0.4 \mathrm{mg} / \mathrm{kg}$ ) reduced to $80 \%$ (5th day), $20 \%$ (10th day), and $0 \%$ (15th day). However, the higher concentration promoted $20 \%$ mortality, and the intermediate concentration promoted $5 \%$ mortality during the course of the study, whereas no mortality was observed for the lower concentration (Figure 7b). For the higher concentration, lethargy and darkening of fish color were also observed. These results are not surprising considering the known relative neurotoxicity of ivermectin. ${ }^{50,51}$ Indeed, previous studies in Atlantic salmon, where $0.5 \mathrm{mg} / \mathrm{kg}$ of ivermectin was administrated every other day sprayed directly over food, reported high mortality over $85 \%$ during 15 days of treatment. $^{52}$ In our study, the mortality was lower for the higher concentration during the same period, which suggests improvement of treatment via drug delivery particles. Moreover, despite oral administration being the most viable route for fish treatment, ${ }^{53}$ one has to consider the fact that distribution of actives in feeding is not proportional between fish, i.e., some may ingest more particles than others, which may have contributed to mortality observed for the higher concentration of ivermectin. Actually, nematode treatment was globally more efficient with $0.2 \mathrm{mg} / \mathrm{kg}$ of ivermectin particles for which low mortality was observed besides complete elimination of parasites in 15 days.

The second experiment was conducted under the same conditions using mebendazole particles and focused on trematode treatment. Figure $7 \mathrm{c}$ shows $100 \%$ prevalence equally for the control and drug-free particles during 15 days, as observed in the first experiment, confirming that particles alone have any effect over fish and parasites. Now, the particles with lower concentration of drug $(0.1 \mathrm{mg} / \mathrm{kg})$ provided a reduction to $80 \%$ prevalence only at the 15 th day; with the intermediate concentration $(0.2 \mathrm{mg} / \mathrm{kg})$, reduction to $80 \%$ was achieved at the 10th day and $60 \%$ at the 15 th day, and the higher concentration $(0.4 \mathrm{mg} / \mathrm{kg})$ provided a better reduction profile to 80,60 , and $40 \%$ at the 5 th, 10 th, and 15 th day, respectively. Of note, the experiment employing mebendazole particles did not cause mortality or lethargy, neither physical aspect and behavior changes, confirming good safety of the drug as described elsewhere. ${ }^{54}$

These latter results may suggest that concentrations of mebendazole were insufficient to completely eliminate the parasites. However, one has to further consider that digenetic trematodes are especially difficult to treat because this class of parasites is generally absent in $\gamma$-amino-butyric acid (GABA), which is a main target of antiparasitic drugs, leading to flaccid paralysis of parasites. ${ }^{55}$ Mebendazole also acts by binding on $\beta$ tubulin molecules, thereby disrupting cell functions such as cell division and transport, which hence may be effective on digenetic trematodes. ${ }^{55}$ However, low treatment yields have been described so far. ${ }^{56}$ 
As a matter of fact, efficacy of treatment obviously depends on efficacy of the drug action. However, the use of particles provides better vectorization, i.e., optimized delivery of the drug to the site of expected action with reduced route degradation. Thereby, results of these experiments provide evidence that the composite particles allowed treatment and showed enhanced yields compared to those of previous studies, suggesting that the composites may be applied as a drug delivery system. Moreover, this is the first in vivo study performed in ornamental fish from the Amazon basin applying composite drug delivery particles for treatment of endoparasites. Hence, direct comparison with results on fish from other species cannot be made. Nevertheless, one emphasizes the importance of treating ornamental fish designed for exportation in the sense that international translocation of parasites may represent a serious concern. ${ }^{57}$ Therefore, if parasites spread and develop in new environments, it may represent a catastrophic environmental menace with outbreak of pathologies and disturbance of natural ecosystems. ${ }^{57-59}$ Importantly, the analyzed Corydoras fish presented high prevalence of $100 \%$ for both endoparasites, which in turn allowed proper evaluation of the particles as drug delivery devices for improved treatment of the gastrointestinal parasites of the fish.

The experiments were conducted during 15 days to optimize fish treatment in a relatively short period of time, aiming to avoid fish suffering and taking into account demands of the ornamental fish industry that require fast treatment, thereby reducing long periods of fish storage, which may increase costs and mortality thus leading to the trade being unprofitable. The number of fish submitted to the experiments was also optimized to minimize fish harm and avoid many and unnecessary necropsies following animal etic procedures.

In terms of particle advantages, the proper composition of particles, made with alginate and chitosan, may have contributed to ingestion of particles because both polysaccharides are components of fish nourishment, e.g., used in the production of commercial fish food for increasing palatability and nutritional yields. ${ }^{60}$ In terms of particle performance, as characterized in the previous section, when placed in the aquarium water of $\mathrm{pH} \sim 6.5$, the same have a negative surface charge, which may provide physical stability during the route of administration. Indeed, when ingested by the fish, particles initially face the buccopharyngeal membranes where $\mathrm{pH}$ ranges around water $\mathrm{pH}$, and thus, the negative surface charge of particles is crucial to reduce mucous interaction in these regions considering the electrostatic repulsion to the negative surface charge of cells. ${ }^{61}$ Hence, this may allow transit of particles and low retention. Thereby, particles reach the stomach where $\mathrm{pH}$ varies around 2.5 in fasting, ${ }^{62}$ which promotes charge reversal to particles. Now with a positive surface charge, particles may interact with stomach mucosa of strong negative charge due the presence of transmembrane mucin glycoproteins. ${ }^{63}$ This scenario may allow prolonged permanence of the particles in the stomach. During food digestion, the $\mathrm{pH}$ can increase to $\sim 4.5{ }^{62}$ Hence, $\mathrm{pH}$ variations and the proper natural digestion process induce slow particle degradation with attenuated release of drugs to the gastrointestinal tract, promoting the final parasite treatment. This ideal performance is certainly not restrictive to fish parasite treatment but may be applied to a plethora of finalities in living beings, including human application, denoting the composite particles as a promising drug delivery system.

\section{CONCLUDING REMARKS}

The production, structure characteristics, and in vivo performance of $\mathrm{pH}$-responsive biocompatible drug delivery particles is described. Intense electrostatic interactions between ionized alginate and protonated chitosan promotes assembly of particles under thermodynamically favored conditions. Inclusion of model drugs of small or large molecular structure does not impede the particle production neither does it spoil their main structural features. The final multifunctionality of the particles relies on structural changes and surface charge changes, which concomitantly respond to $\mathrm{pH}$ variation. Hydration of large chains of the alginate polysaccharide leads to an increase in hydration volume, which thus increments the hydrodynamic diameter of the particles producing swollen expanded structures at $\mathrm{pH}$ 2.50. The further ionization of alginate at $\mathrm{pH}$ levels between 4.10 and 4.50 leads to charge reversal and compaction of particles under strong interaction with protonated chitosan. Moreover, deprotonation of chitosan at $\mathrm{pH} 6.50$ prompts ionized alginate self-repulsion, producing new swelling of the particle structure. The particles are thus $\mathrm{pH}$ structure responsive in the sense of swelling or compressing at the same time the surface charge adjusts to the $\mathrm{pH}$ condition, which may generate advantageous physical stability and charge antagonism with cells during the route of administration with interaction activity that may be induced at the intended drug delivery target.

The efficacy of the particles was proven with incorporation of two model antiparasitic drugs and in vivo application as oral administration in highly parasitized Corydoras fish. The in vivo performance denotes the effective action of the particles in treatment of the parasite infection of fish, highlighting the $\mathrm{pH}$ responsive devices as prominent biomaterials of real applications in biological, biomedical, and environmental fields.

\section{ASSOCIATED CONTENT}

\section{S Supporting Information}

The Supporting Information is available free of charge on the ACS Publications website at DOI: 10.1021/acs.biomac.7b01630.

Data relative to mass loss in particle preparation (Table S1) and DLS data for chitosan and alginate (Table S2) (PDF)

\section{AUTHOR INFORMATION}

\section{Corresponding Authors}

*E-mail: patrickmathews83@gmail.com.

*E-mail: mertins@unifesp.br. Tel.: +55 115576 2338. Fax: +55

1155715780.

ORCID 8

Omar Mertins: 0000-0002-5028-9215

Notes

The authors declare no competing financial interest.

\section{ACKNOWLEDGMENTS}

The authors thank the Brazilian Synchrotron Light Laboratory (LNLS) for allowing SAXS experiments and Prof. Maria A. Juliano for providing and supporting lyophilization of samples. The study was supported by Sao Paulo Research Foundation, FAPESP, through research grants 2015/23948-5 and 2016/ 13368-4. A.C.M.F.P. also thanks FAPESP for MSc fellowship (process: 2017/16722-6). 


\section{REFERENCES}

(1) Xu, W.; Ding, J.; Chen, X. Reduction-responsive polypeptide micelles for intracellular delivery of antineoplastic agent. Biomacromolecules 2017, 18, 3291-3301.

(2) Silva, A. S.; Tavares, M. T.; Aguiar-Ricardo, A. Sustainable strategies for nano-in-micro particle engineering for pulmonary delivery. J. Nanopart. Res. 2014, 16, 2602.

(3) Mertins, O.; Mathews, P. D.; Gomide, A. B.; Baptista, M. S.; Itri, R. Effective protection of biological membranes against photooxidative damage: polymeric antioxidant forming a protecting shield over the membrane. Biochim. Biophys. Acta, Biomembr. 2015, 1848, $2180-2187$.

(4) Benelli, G. Gold nanoparticles - against parasites and insect vectors. Acta Trop. 2018, 178, 73-80.

(5) Andreeva, T. D.; Stoichev, S.; Taneva, S. G.; Krastev, R. Hybrid graphene oxide/polysaccharide nanocomposites with controllable surface properties and biocompatibility. Carbohydr. Polym. 2018, $181,78-85$.

(6) Kiew, S. F.; Kiew, L. V.; Lee, H. B.; Imae, T.; Chung, L. Y. Assessing biocompatibility of graphene oxide-based nanocarriers: a review. J. Controlled Release 2016, 226, 217-228.

(7) Macgregor, R. R.; Graziani, A. L. Oral administration of antibiotics: a rational alternative to the parenteral route. Clin. Infect. Dis. 1997, 24, 457-467.

(8) Gamboa, J. M.; Leong, K. W. In vitro and in vivo models for the study of oral delivery of nanoparticles. Adv. Drug Delivery Rev. 2013, 65, 800-810.

(9) Colombo, P.; Sonvico, F.; Colombo, G.; Bettini, R. Novel platforms for oral drug delivery. Pharm. Res. 2009, 26, 601-611.

(10) Han, M.; Fang, Q. L.; Zhan, H. W.; Luo, T.; Liang, W. Q.; Gao, J. Q. In vitro and in vivo evaluation of a novel capsule for colonspecific drug delivery. J. Pharm. Sci. 2009, 98, 2626-2635.

(11) Liu, L.; Yao, W. D.; Rao, Y. F.; Lu, X. Y.; Gao, J. Q. pHResponsive carriers for oral drug delivery: challenges and opportunities of current platforms. Drug Delivery 2017, 24, 569-581.

(12) Rinaudo, M. Chitin and chitosan: properties and applications. Prog. Polym. Sci. 2006, 31, 603-632.

(13) Zargar, V.; Asghari, M.; Dashti, A. A review on chitin and chitosan polymers: structure, chemistry, solubility, derivatives, and applications. ChemBioEng Rev. 2015, 2, 204-226.

(14) Lee, K. Y.; Mooney, D. J. Alginate: properties and biomedical applications. Prog. Polym. Sci. 2012, 37, 106-126.

(15) Stana, J.; Stergar, J.; Gradišnik, L.; Flis, V.; Kargl, R.; Fröhlich, E.; Kleinschek, K. S.; Mohan, T.; Maver, U. Multilayered polysaccharide nanofilms for controlled delivery of pentoxifylline and possible treatment of chronic venous ulceration. Biomacromolecules 2017, 18, 2732-2746.

(16) Wang, F.; Yang, S.; Yuan, J.; Gao, Q.; Huang, C. Effective method of chitosan-coated alginate nanoparticles for target drug delivery applications. J. Biomater. Appl. 2016, 31, 3-12.

(17) Bagre, A. P.; Jain, K.; Jain, N. K. Alginate coated chitosan core shell nanoparticles for oral delivery of enoxaparin: in vitro and in vivo assessment. Int. J. Pharm. 2013, 456, 31-40.

(18) Mukhopadhyay, P.; Chakraborty, S.; Bhattacharya, S.; Mishra, R; Kundu, P. P. pH-Sensitive chitosan/alginate core-shell nanoparticles for efficient and safe oral insulin delivery. Int. J. Biol. Macromol. 2015, 72, 640-648.

(19) Daly, M. M.; Knorr, D. Chitosan-alginate complex coacervate capsules: effects of calcium chloride, plasticizers, and polyelectrolytes on mechanical stability. Biotechnol. Prog. 1988, 4, 76-81.

(20) Baruch, L.; Machluf, M. Alginate-chitosan complex coacervation for cell encapsulation: effect on mechanical properties and on longterm viability. Biopolymers 2006, 82, 570-579.

(21) Motwani, S. K.; Chopra, S.; Talegaonkar, S.; Kohli, K.; Ahmad, F. J.; Khar, R. K. Chitosan-sodium alginate nanoparticles as submicroscopic reservoirs for ocular delivery: formulation, optimization and in vitro characterization. Eur. J. Pharm. Biopharm. 2008, 68, $513-525$.
(22) Kasim, N. A.; Whitehouse, M.; Ramachandran, C.; Bermejo, M.; Lennernäs, H.; Hussain, A. S. Molecular properties of WHO essential drugs and provisional biopharmaceutical classification. Mol. Pharmaceutics 2004, 1, 85-96.

(23) Mertins, O.; Sebben, M.; Pohlmann, A. R.; da Silveira, N. P. Production of soybean phosphatidylcholine-chitosan nanovesicles by reverse phase evaporation: a step by step study. Chem. Phys. Lipids 2005, 138, 29-37.

(24) Mertins, O.; Dimova, R. Insights on the interactions of chitosan with phospholipid vesicles. Part II: membrane stiffening and pore formation. Langmuir 2013, 29, 14552-14559.

(25) Mathews, P. D.; Naldoni, J.; Adriano, E. A. Morphology and small subunit rDNA-based phylogeny of a new Henneguya species, infecting the ornamental fish Corydoras leucomelas from the Peruvian Amazon. Acta Trop. 2017, 176, 51-57.

(26) Ali, M.; Afzal, M.; Verma, M.; Misra-Bhattacharya, S.; Ahmad, F. J.; Dinda, A. K. Improved antifilarial activity of ivermectin in chitosanalginate nanoparticles against human lymphatic filarial parasite. Parasitol. Res. 2013, 112, 2933-2943.

(27) Mathews, P. D.; Mertins, O. Dispersion of chitosan in liquid crystalline lamellar phase: production of biofriendly hydrogel of nano cubic topology. Carbohydr. Polym. 2017, 157, 850-857.

(28) Stoeckel, D.; Wallacher, D.; Zickler, G. A.; Perlich, J.; Tallarek, U.; Smarsly, B. M. Coherent analysis of disordered mesoporous adsorbents using small angle $\mathrm{X}$-ray scattering and physisorption experiments. Phys. Chem. Chem. Phys. 2014, 16, 6583-6592.

(29) de Souza, C. F. V.; Faccin, D. J. L.; Mertins, O.; Heck, J. X.; da Silveira, N. P.; Secchi, A. R.; Ayub, M. A. Z. Kinetics of thermal inactivation of transglutaminase from a newly isolated Bacillus circulans BL32. J. Chem. Technol. Biotechnol. 2009, 84, 1567-1575.

(30) Mertins, O. Estudos fisico-quimicos e estruturais de lipossomas compósitos de fosfatidilcolina e quitosana. Ph.D. Thesis, Universidade Federal do Rio Grande do Sul, Brazil, 2008. http://hdl.handle.net/ $10183 / 14354$

(31) Berne, B. J.; Pecora, R. Dynamic Light Scattering: with applications to Chemistry, Biology, and Physics; Dover Publications: New York, 2000; p 376.

(32) Mathews, P. D.; Silva, M. R. M.; Maia, A. A. M.; Adriano, E. A. Ultrastructure and ssrRNA sequencing of Myxidium amazonense $\mathrm{n}$. sp. a myxosporean parasite of Corydoras melini from the Rio Negro river, Amazonas state. Parasitol. Res. 2015, 114, 4675-4683.

(33) Espinoza, L. L.; Mertins, O.; Gama, G. S.; Patta, A. C. M. F.; Mathews, P. D. A new Myxidium species (Myxozoa: Myxosporea) infecting the gallbladder of the turtle Podocnemis unifilis (Testudines: Podocnemididae) from Peruvian Amazon. Acta Trop. 2017, 172, 7579.

(34) Vázquez, N. D.; Mathews, P. D.; Chu-Koo, F. W.; Martín, S. T.; Orbe, R. I. Fauna parasitaria de juveniles de arahuana, Osteoglossum bicirrhosum (Vandelli, 1829) cultivado en el Centro de Investigaciones de Quistocoha, Loreto, Perú. Folia Amazonica 2007, 16, 29-33.

(35) Mathews, P. D.; Mathews, J. P. D.; Orbe, R. I. Parasitic infections in juveniles of Prochilodus nigricans kept in semi-intensive fish farm in the Peruvian Amazon. Bull. Eur. Ass. Fish Pathol. 2013, 33, $28-32$.

(36) Mertins, O.; Dimova, R. Binding of chitosan to phospholipid vesicles studied with isothermal titration calorimetry. Langmuir 2011, 27, 5506-5515.

(37) Ma, P. L.; Lavertu, M.; Winnik, F. M.; Buschmann, M. D. New insights into chitosan DNA interactions using isothermal titration microcalorimetry. Biomacromolecules 2009, 10, 1490-1499.

(38) Mertins, O.; Dimova, R. Insights on the interactions of chitosan with phospholipid vesicles. Part I: effect of polymer deprotonation. Langmuir 2013, 29, 14545-14551.

(39) Rinaudo, M.; Pavlov, G.; Desbrieres, J. Solubilization of chitosan in strong acid medium. Int. J. Polym. Anal. Charact. 1999, 5, 267-276.

(40) Rinaudo, M.; Pavlov, G.; Desbrieres, J. Influence of acetic acid concentration on the solubilization of chitosan. Polymer 1999, 40, $7029-7032$. 
(41) Mertins, O.; Schneider, P. H.; Pohlmann, A. R.; da Silveira, N. P. Interaction between phospholipids bilayer and chitosan in liposomes investigated by 31P NMR spectroscopy. Colloids Surf., B 2010, 75, 294-299.

(42) Baños, F. G. D.; Peña, A. I. D.; Cifre, J. G. H.; Martínez, M. C. L.; Ortega, A.; Torre, J. G. Influence of ionic strength on the flexibility of alginate studied by size exclusion chromatography. Carbohydr. Polym. 2014, 102, 223-230.

(43) ITC data analysis in Origin. In Tutorial Guide, 7th ed.; MicroCal LLC: Northampton, MA, 2004.

(44) Smidsrod, O.; Glover, R. M.; Whittington, S. G. The relative extension of alginates having different chemical composition. Carbohydr. Res. 1973, 27, 107-118.

(45) Christensen, B. E.; Vold, I. M. N.; Vårum, K. M. Chain stiffness and extension of chitosans and periodate oxidised chitosans studied by size-exclusion chromatography combined with light scattering and viscosity detectors. Carbohydr. Polym. 2008, 74, 559-565.

(46) Schatz, C.; Viton, C.; Delair, T.; Pichot, C.; Domard, A. Typical physicochemical behaviors of chitosan in aqueous solution. Biomacromolecules 2003, 4, 641-648.

(47) Porod, G. In Small Angle X-ray Scattering; Glatter, O., Kratky, O., Eds.; Academic Press: London, 1982; pp 17-51.

(48) Surnar, B.; Jayakannan, M. Stimuli-responsive poly(caprolactone) vesicles for dual drug delivery under the gastrointestinal tract. Biomacromolecules 2013, 14, 4377-4387.

(49) Neal, M. J. Medical pharmacology at a glance, 8th ed.; John Wiley \& Sons, Ltd., 2016.

(50) Høy, T.; Horsberg, T. E.; Nafstad, I. The disposition of ivermectin in Atlantic salmon (Salmo salar). Pharmacol. Toxicol. 1990, 67, 307-312.

(51) Horsberg, T. E. Avermectin use in aquaculture. Curr. Pharm. Biotechnol. 2012, 13, 1095-1102.

(52) Johnson, S. C.; Kent, M. L.; Whitaker, D. J.; Margolis, L. Toxicity and pathological effects of orally administered ivermectin in Atlantic, chinook, and coho salmon and steelhead trout. Dis. Aquat. Org. 1993, 17, 107-112.

(53) Ji, J.; Torrealba, D.; Ruyra, A.; Roher, N. Nanodelivery systems as new tools for immunostimulant or vaccine administration: targeting the fish immune system. Biology 2015, 4, 664-696.

(54) Chagas, E. C.; Araújo, L. D.; Martins, M. L.; Gomes, L. C.; Malta, J. C. O.; Varella, A. B.; Jerônimo, G. T. Mebendazole dietary supplementation controls Monogenoidea (Platyhelminthes: Dactylogyridae) and does not alter the physiology of the freshwater fish Colossoma macropomum (Cuvier, 1818). Aquaculture 2016, 464, 185189.

(55) Martin, R. J. Modes of action of anthelmintic drugs. Vet. J. 1997, 154, 11-34.

(56) Reimschuessel, R.; Gieseker, C.; Poynton, S. In vitro effect of seven antiparasitics on Acolpenteron ureteroecetes (Dactylogyridae) from largemouth bass Micropterus salmoides (Centrarchidae). Dis. Aquat. Org. 2011, 94, 59-72.

(57) Whittington, R.; Chong, R. Global trade in ornamental fish from an Australian perspective: the case for revised import risk analysis and management strategies. Prev. Vet. Med. 2007, 81, 92-116.

(58) Camus, A. C.; Dill, J. A.; McDermott, A. J.; Clauss, T. M.; Berliner, A. L.; Boylan, S. M.; Soto, E. Francisella noatunensis subsp. orientalis infection in Indo-Pacific reef fish entering the United States through the ornamental fish trade. J. Fish Dis. 2013, 36, 681-684.

(59) Camus, A. C.; Dill, J. A.; Rosser, T. G.; Pote, L. M.; Griffin, M. J. Myxobolus axelrodi nsp. (Myxosporea: Myxobolidae) a parasite infecting the brain and retinas of the cardinal tetra Paracheirodon axelrodi (Teleostei: Characidae). Parasitol. Res. 2017, 116, 387-397.

(60) Volpe, M. G.; Varricchio, E.; Coccia, E.; Santagata, G.; Di Stasio, M.; Malinconico, M.; Paolucci, M. Manufacturing pellets with different binders: effect on water stability and feeding response in juvenile Cherax albidus. Aquaculture 2012, 324-325, 104-110.

(61) McLaughlin, S. The electrostatic properties of membrane. Annu. Rev. Biophys. Biophys. Chem. 1989, 18, 113-136.
(62) Steffens, W. Principles of fish nutrition; Ellis Horwood: London, 1989; p 235.

(63) Pérez-Sánchez, J.; Estensoro, I.; Redondo, M. J.; Calduch-Giner, J. A.; Kaushik, S.; Sitjà-Bobadilla, A. Mucins as diagnostic and prognostic biomarkers in a fish-parasite model: transcriptional and functional analysis. PLoS One 2013, 8, e65457. 


\section{Supporting Information for}

\section{Targeted drug delivery and treatment of endoparasites with biocompatible particles of $\mathrm{pH}$ responsive structure}

Patrick D. Mathews ${ }^{a^{*}}$, Ana C. M. Fernandes Patta ${ }^{a}$, Joao V. Gonçalves ${ }^{a}$, Gabriella dos Santos Gama ${ }^{a}$, Irene T. S. Garcia ${ }^{b}$, Omar Mertins $^{a^{*}}$

a Department of Biophysics, Paulista School of Medicine, Federal University of Sao Paulo, Sao Paulo 04023-062, Brazil

${ }^{b}$ Department of Physical-Chemistry, Institute of Chemistry, Federal University of Rio Grande do Sul, Porto Alegre 91501-970, Brazil

*Address correspondence to Patrick D. Mathews and Omar Mertins

E-mails: patrickmathews83@gmail.com; mertins@unifesp.br 


\section{Supporting Information}

Table S1. Initial masses used in preparation of triplicates (1-3) of alginate/chitosan particles free of drug (ALGCH), in presence of $38 \mu \mathrm{M}$ mebendazole (MB) or $38 \mu \mathrm{M}$ ivermectin (IVM); recovered masses obtained after lyophilization of supernatants in the particles washing procedure and corresponding averages with standard deviation.

\begin{tabular}{cccc}
\hline Sample & $\begin{array}{c}\text { Initial mass } \\
(\mathbf{m g})\end{array}$ & $\begin{array}{c}\text { Recovered mass } \\
(\mathbf{m g})\end{array}$ & $\begin{array}{c}\text { Average of recovered masses } \\
(\mathbf{m g})\end{array}$ \\
\hline ALGCH (1) & 100.00 & 13.81 & $15.93 \pm 2.22$ \\
\hline ALGCH (2) & 100.00 & 15.73 & \\
\hline ALGCH (3) & 100.00 & 18.24 & $15.77 \pm 1.75$ \\
\hline ALGCH-MB (1) & 111.21 & 14.26 & \\
\hline ALGCH-MB (2) & 111.21 & 15.35 & \\
\hline ALGCH-MB (3) & 111.21 & 17.69 & \\
\hline ALGCH-IVM (1) & 133.25 & 12.93 & \\
\hline ALGCH-IVM (2) & 133.25 & 17.72 & \\
\hline ALGCH-IVM (3) & 133.25 & 18.51 & Total average: $16.03 \mathrm{mg}$ \\
\hline
\end{tabular}

Table S2. Hydrodynamic diameters (Dh, $\mathrm{nm}$ ) obtained by DLS for $0.1 \mathrm{mg} / \mathrm{mL}$ solutions of chitosan $(\mathrm{CH})$ and alginate (ALG) in different buffers $(80 \mathrm{mM})$ at $25^{\circ} \mathrm{C}$. Maximal $\mathrm{pH}$ variation was 0.02. Dh are average of five independent measurements and variations are standard deviation. Aggregates represent particles size over $2 \mu \mathrm{m}$.

\begin{tabular}{|ccccccc|}
\hline pH & $\mathbf{2 . 5 0}$ & $\mathbf{3 . 7 9}$ & $\mathbf{4 . 1 0}$ & $\mathbf{4 . 3 2}$ & $\mathbf{4 . 5 0}$ & $\mathbf{6 . 5 0}$ \\
\hline CH Dh & $41 \pm 18$ & $47 \pm 26$ & $58 \pm 17$ & $52 \pm 23$ & $71 \pm 15$ & aggregates \\
\hline ALG Dh & aggregates & $241 \pm 78$ & $119 \pm 51$ & $137 \pm 42$ & $108 \pm 47$ & $87 \pm 32$ \\
\hline
\end{tabular}

